\title{
AN OPTIMAL PORTFOLIO PROBLEM IN A DEFAULTABLE MARKET
}

\author{
LIJUN BO, ${ }^{*}$ Xidian University \\ YONGJIN WANG ${ }^{* *}$ AND \\ XUEWEI YANG, ${ }^{* * *}$ Nankai University
}

\begin{abstract}
We consider a portfolio optimization problem in a defaultable market. The investor can dynamically choose a consumption rate and allocate his/her wealth among three financial securities: a defaultable perpetual bond, a default-free risky asset, and a money market account. Both the default risk premium and the default intensity of the defaultable bond are assumed to rely on some stochastic factor which is described by a diffusion process. The goal is to maximize the infinite-horizon expected discounted log utility of consumption. We apply the dynamic programming principle to deduce a HamiltonJacobi-Bellman equation. Then an optimal Markov control policy and the optimal value function is explicitly presented in a verification theorem. Finally, a numerical analysis is presented for illustration.
\end{abstract}

Keywords: Portfolio optimization; defaultable security; stochastic factor; HamiltonJacobi-Bellman equation; sub/super-solution

2010 Mathematics Subject Classification: Primary 93E20; 60H30

\section{Introduction}

Stochastic portfolio optimization has been an attractive topic in the subject of mathematical finance. For the investment problem in a stock and a riskless bond, the pioneering works by Merton [19], [20], [21, pp. 95-212] first approached the strategy for maximizing the total expected discounted utility of consumption. From then on, various default-free optimal investment models have been proposed and investigated in the literature (see, e.g. [6], [11], [17], [22], and [23]). Among them, Fleming and Pang [11] discussed a classical Merton portfolio optimization problem, where the interest rate is assumed to fluctuate from time to time. The objective was to maximize the expected discounted HARA utility of consumption at the infinite horizon. In a subsequent paper, Pang [22] treated the analogue problem with log utility. Pham [23] studied an optimal investment problem, in which the instantaneous rate and the volatility are assumed to rely on a stochastic factor that is described by a Markov diffusion process, and the goal is to maximize the expected HARA utility of the terminal wealth.

Recently, the optimal investment and hedging with the defaultable claims have aroused much more attention (see, e.g. [1], [2], [3], [4], [14], [16], and [18]). Hou and Jin [16] studied an optimal investment problem with default risk under the conditional diversification assumption (which implies an asymptotic disappearance of the jump-risk premium), as in [15]. Dynamics

Received 13 July 2009; revision received 17 April 2010.

* Postal address: Department of Mathematics, Xidian University, Xi'an 710071, P. R. China.

** Postal address: School of Business, Nankai University, Tianjin 300071, P. R. China.

*** Postal address: School of Mathematical Sciences and TEDA Institute of Computational Finance, Nankai University, Tianjin 300071, P. R. China. Email address: xwyangnk@yahoo.com.cn 
with constant default risk premium and constant default intensity for the price process of a defaultable bond was proposed in [14]. Bielecki and Jang [4] established an optimal asset allocation for maximizing the expected HARA utility of the terminal wealth.

In this paper we consider a stochastic portfolio optimization problem in a reduced-form defaultable market. The investor can dynamically choose a consumption rate and allocate his/her wealth among three financial securities: a defaultable perpetual bond, a default-free risky asset, and a money market account. Both the default risk premium and the default intensity of the defaultable bond are assumed to rely on some stochastic factor which is described by a diffusion process. (This assumption seems to be reasonable, and has been adopted in some known references; see, e.g. [23]. In fact, the stochastic differential equations in which the characteristic parameters depend on economic factors (state variables) are often used in finance to model different types of economic phenomenon, such as the random fluctuant interest rate and stochastic volatility; see, e.g. [10] and [11]. It is also natural to allow the default intensity and the default risk premium to depend on economic factors; see, e.g. Chapter 11 of [8] and the references therein.) Here we intend to maximize the infinite-horizon expected discounted $\log$ utility of consumption. For this purpose, we apply the dynamic programming principle to deduce a Hamilton-Jacobi-Bellman (HJB) equation, and then the sub/super-solution technique for partial differential equations (see, e.g. [11]) is adopted to study the solution of the HJB equation. The optimal Markov control policy and the optimal value function is explicitly presented in a verification theorem. Finally, we present a numerical analysis of the optimal control strategy and the value function.

The paper is organized as follows. In Section 2 we present the price dynamics of financial securities. The HJB equation for the optimal argument with a defaultable security is deduced and investigated in Section 3. Section 4 is devoted to proving a verification theorem. In Appendix A we present the derivation of the price dynamics for the perpetual defaultable bond with constant market parameters. In Appendix B we present a parametric sensitivity analysis on the optimal control strategy and the value function.

\section{Price dynamics of financial securities}

In this paper we consider an investor who dynamically allocates his/her wealth among a defaultable perpetual bond, a default-free risky asset, and a money market account. The main task in this section is to describe the price dynamics of the above three financial securities.

Let $(\Omega, \mathcal{F}, \mathrm{P})$ be a complete real-world probability space, and let $\tau$ be a nonnegative random variable on this space. For $t \geq 0$, define a default indicator process $\left(z_{t}\right)_{t \geq 0}$ by

$$
z_{t}=\mathbf{1}_{\{\tau \leq t\}}
$$

Suppose that $\left(W_{t}, \breve{W}_{t}\right)_{t \geq 0}$ is a two- dimensional standard Brownian motion on $(\Omega, \mathcal{F}, \mathrm{P})$, and let $\mathbb{F}=\left(\mathcal{F}_{t}\right)_{t \geq 0}$ be the augmented natural filtration of $\left(W_{t}, \breve{W}_{t}\right)_{t \geq 0}$. Let $\mathscr{D}_{t}=\sigma\left(z_{u} ; 0 \leq u \leq t\right)$ and $g_{t}=\mathscr{F}_{t} \vee \mathscr{D}_{t}$ with $t \geq 0$. Then $\tau$ is a $\mathbb{G}=\left(g_{t}\right)_{t \geq 0}$ stopping time. We assume that all the filtrations satisfy the usual hypotheses of completeness and right continuity.

We suppose that $\tau$ has a positive $\mathbb{F}$-adapted intensity process $\left(\lambda_{t}\right)_{t \geq 0}$. (This implies that $\tau$ is a totally inaccessible $\mathbb{G}$-stopping time; see, e.g. Section VI.78 of [7]). Now

$$
m_{t}:=z_{t}-\int_{(0, t \wedge \tau]} \lambda_{s} \mathrm{~d} s=z_{t}-\int_{(0, t]}\left(1-z_{s}\right) \lambda_{s} \mathrm{~d} s, \quad t \geq 0,
$$


is a $(\mathrm{P}, \mathbb{G})$-martingale. Accordingly, for any $t>0$, the survival probability

$$
\mathrm{P}(\tau>t)=\mathrm{E}\left[\mathrm{E}\left[1-z_{t} \mid \mathscr{F}_{t}\right]\right]=\mathrm{E}\left[\exp \left(-\int_{0}^{t} \lambda_{s} \mathrm{~d} s\right)\right] .
$$

Herein, we suppose that the nonnegative intensity process $\left(\lambda_{t}\right)_{t \geq 0}$ and the default risk premium $\left(1 / \eta_{t}\right)_{t \geq 0}$ rely on an $\mathbb{F}$-adapted stochastic economic factor $\left(y_{t}\right)_{t \geq 0}$ (see, e.g. [23]), i.e. there exist a nonnegative measurable function $\lambda(\cdot)$ on $\mathbb{R}^{+}$and a $(0,1]$-valued measurable function $\eta(\cdot)$ such that

$$
\lambda_{t}=\lambda\left(y_{t}\right), \quad \eta_{t}=\eta\left(y_{t}\right), \quad t \geq 0,
$$

where the $\left(y_{t}\right)_{t \geq 0}$ is described by

$$
\mathrm{d} y_{t}=\mu\left(y_{t}\right) \mathrm{d} t+\mathrm{d} \breve{W}_{t}, \quad t>0, y_{0}=y .
$$

Remark 2.1. We suppose that the economic factor is independent of the default-free risky asset in (2.6), below. If the economic factor is correlated with the default-free risky asset, there will be an additional mixed partial derivative term in the HJB equation (3.3), below, and this term dose not have the essential effect on the problem (see, e.g. [11]).

Let $\rho \in(0,1)$ denote the constant loss rate when a default occurs. Here we adopt the 'recovery of market value' scheme, which means that at the default time the bond loses a fraction $\rho$ of its value (see, e.g. Equation (9) of [9]). Then we can suggest price dynamics $\left(p_{t}\right)_{t \geq 0}$ for a defaultable perpetual bond that pays a constant coupon $\widetilde{C}$ per unit time as follows:

$$
\mathrm{d} p_{t}=r p_{t} \mathrm{~d} t+\rho \lambda_{t} p_{t}\left(1-z_{t}\right)\left(\frac{1}{\eta_{t}}-1\right) \mathrm{d} t-\left(1-z_{t}\right) \widetilde{C} \mathrm{~d} t-\rho p_{t-} \mathrm{d} m_{t},
$$

where $\left(m_{t}\right)_{t \geq 0}$ is the $(\mathrm{P}, \mathbb{G})$-martingale defined in (2.2) and $r \in \mathbb{R}^{+}$is the constant interest rate. (The derivation of the dynamics when the market parameters are constant is given in Appendix A. Note that Example 2.2.4 of [5] indicates that $\rho<1$ is possible. Here we directly randomize the market parameters in the dynamics similarly to the method used for the stochastic volatility model (see, e.g. Heston [12]). One must be careful when the market parameters are random, since then the derivation in Appendix A will be not valid.) Moreover, the money market account $\left(\beta_{t}\right)_{t \geq 0}$ and the default-free risky asset $\left(\gamma_{t}\right)_{t \geq 0}$ are described by the price dynamics

$$
\begin{gathered}
\mathrm{d} \beta_{t}=r \beta_{t} \mathrm{~d} t, \quad \beta_{0}=1, \\
\mathrm{~d} \gamma_{t}=b \gamma_{t} \mathrm{~d} t+a \gamma_{t} \mathrm{~d} W_{t}, \quad \gamma_{0}=\gamma>0,
\end{gathered}
$$

where the coefficients $a$ and $b$ are constants. (The reasons for assuming a constant interest rate are two-fold. First, we are mainly concerned with the influence of the default risk in the portfolio argument, so we do not pay much attention to the interest rate risk. Second, the default-free portfolio arguments with the stochastic interest rate have been investigated in [11] and [22]. The context of this paper may be extended to the stochastic interest rate case, but some additional technique should be adopted and the respective argument will probably be more complicated.)

We make the following technical assumptions.

(H1) $\mu(y) \in C^{1}(\mathbb{R})$ and there exist constants $\underline{C}, \bar{C}$ such that $\underline{C} \leq \mu_{y}(y):=\mathrm{d} \mu(y) / \mathrm{d} y \leq \bar{C}$ for all $y \in \mathbb{R}$. 
(H2) There exist constants $C>0$ and $\delta \geq 1$ such that

$$
\lambda(y) \leq C+C|y|^{\delta}, \quad y \in \mathbb{R} .
$$

(H3) The quantity $\eta_{m}:=\inf _{y \in \mathbb{R}} \eta(y)$ is strictly positive.

We conclude this section with the following lemma.

Lemma 2.1. Under assumption (H1), (2.4) admits a unique strong solution $\left(y_{t}\right)_{t \geq 0}$ such that, for some $\alpha_{m}>2 m \bar{C}(m \geq 1)$,

$$
\lim _{T \rightarrow \infty} \mathrm{e}^{-\alpha_{m} T} \mathrm{E}\left[y_{T}^{2 m}\right]=0, \quad \lim _{T \rightarrow \infty} \mathrm{e}^{-\alpha_{m} T} \mathrm{E}\left[\int_{0}^{T} y_{s}^{2 m} \mathrm{~d} s\right]=0 .
$$

Proof. Note that, by $(\mathrm{H} 1)$, the $\operatorname{drift} \mu(\cdot)$ is globally Lipschitzian on $\mathbb{R}$. Then the conclusion follows from Lemma 3.2 of [22].

\section{The optimal portfolio problem with a defaultable perpetual bond}

In this section we explore the stochastic portfolio problem with a defaultable perpetual bond. The goal is to maximize the infinite-horizon expected discounted log utility of consumption.

For each time $t \geq 0$, let $x_{t}$ be the wealth at time $t$, and let $\kappa_{t}$ and $\ell_{t}$ be the respective proportions of wealth $x_{t}$ in the defaultable bond $\left(p_{t}\right)_{t \geq 0}$ and the default-free risky asset $\left(\gamma_{t}\right)_{t \geq 0}$. Then $1-\kappa_{t}-\ell_{t}$ is the $t$-time proportion of wealth $x_{t}$ in the money market account $\left(\beta_{t}\right)_{t \geq 0}$. In addition, the investor can choose a consumption rate $c_{t}>0$ at time $t \geq 0$. Suppose that the initial wealth $x_{0}>0$. Then, by virtue of the self-financing investment policy (see, e.g. [14]), the dynamics of the wealth process $\left(x_{t}\right)_{t \geq 0}$ follow

$\mathrm{d} x_{t}=\frac{\left(1-\kappa_{t}-\ell_{t}\right) x_{t}}{\beta_{t}} \mathrm{~d} \beta_{t}+\frac{\kappa_{t} x_{t-}}{p_{t-}} \mathrm{d} p_{t}+\frac{\ell_{t} x_{t}}{\gamma_{t}} \mathrm{~d} \gamma_{t}+\frac{\kappa_{t} x_{t}}{p_{t}}\left(1-z_{t}\right) \widetilde{C} \mathrm{~d} t-c_{t} x_{t} \mathrm{~d} t, \quad t>0$.

Now summarizing (2.5), (2.6), and (3.1), we have the wealth dynamics

$$
\begin{aligned}
\frac{\mathrm{d} x_{t}}{x_{t-}}= & r\left(1-\ell_{t}\right) \mathrm{d} t+b \ell_{t} \mathrm{~d} t+\rho \kappa_{t}\left(1-z_{t}\right) \lambda\left(y_{t}\right)\left(\frac{1}{\eta\left(y_{t}\right)}-1\right) \mathrm{d} t-c_{t} \mathrm{~d} t+a \ell_{t} \mathrm{~d} W_{t} \\
& -\rho \kappa_{t-}\left(1-z_{t-}\right) \mathrm{d} m_{t}, \quad t>0 .
\end{aligned}
$$

Remark 3.1. The difference between our analysis and Equation (4.5.14) of [14, Chapter 4] (or Lemma 3 of [4]) is that the default process $\left(\lambda_{t}\right)_{t \geq 0}$ and the default risk premium $\left(1 / \eta_{t}\right)_{t \geq 0}$ rely on a stochastic factor process (which has been assumed for some default-free stochastic portfolio optimization arguments; see, e.g. [10] and [11]), rather than on two constants.

Our aim is to seek an optimal allocation $\left(\kappa_{t}, \ell_{t}\right)_{t \geq 0}$ and an optimal consumption rate $\left(c_{t}\right)_{t \geq 0}$ for the wealth $\left(x_{t}\right)_{t \geq 0}$ to maximize the infinite-horizon expected discounted utility of consumption. To this aim, we restrict the allocation $\left(\kappa_{t}, \ell_{t}\right)_{t \geq 0}$ and the consumption rate $\left(c_{t}\right)_{t \geq 0}$ to some admissible control set $\mathcal{A}(\mathbb{G})$.

Definition 3.1. A càdlàg $\mathbb{G}$-adapted Markov control $\left(\kappa_{t}, \ell_{t}, c_{t}\right)_{t \geq 0}$ is in the admissible control space $\mathcal{A}(\mathbb{G})$ if the following conditions hold:

$$
\kappa_{t} \in\left[0, \frac{1}{\rho}\right), \quad \ell_{t} \in \mathbb{R}, \quad c_{t}>0, \quad \text { for all } t \geq 0 .
$$


Remark 3.2. In Definition 3.1, the restriction $\kappa . \in\left[0,1 / \rho\right.$ ) (which ensures that $x_{t}>0$ for all $t \geq 0$ ) is the so-called avoiding bankruptcy condition (see Remark 5.2.4 of [14]), and each element of the admissible control space $\mathcal{A}(\mathbb{G})$ is a bankruptcy avoiding portfolio.

We consider the $\log$ utility function on $(0, \infty)$, i.e. $U(x)=\log x, x>0$.

Remark 3.3. The log utility function $U(x)$ corresponds to the HARA utility function in the limiting case of relative risk aversion equal to 1 .

Let $\alpha>0$ be a discount factor. For an admissible control $\left(\kappa_{t}, \ell_{t}, c_{t}\right)_{t \geq 0}$ and an initial value $(x, y, z) \in(0, \infty) \times \mathbb{R} \times\{0,1\}$, define the objective functional on an infinite time horizon by

$$
\begin{aligned}
J(x, y, z, \kappa ., \ell ., c .) & =\mathrm{E}_{x, y, z}\left[\int_{0}^{\infty} \mathrm{e}^{-\alpha t} U\left(c_{t} x_{t}\right) \mathrm{d} t\right] \\
& :=\mathrm{E}\left[\int_{0}^{\infty} \mathrm{e}^{-\alpha t} U\left(c_{t} x_{t}\right) \mathrm{d} t \mid x_{0}=x, y_{0}=y, z_{0}=z\right] .
\end{aligned}
$$

Our goal is to maximize the objective functional $J(x, y, z, \kappa ., \ell ., c$.) for all admissible $\left(\kappa_{t}, \ell_{t}, c_{t}\right)_{t \geq 0}$. The value function is given by

$$
v(x, y, z):=\sup _{(\kappa ., \ell ., c .) \in \mathcal{A}(\mathbb{G})} J(x, y, z, \kappa ., \ell ., c .)
$$

for each $(x, y, z) \in(0, \infty) \times \mathbb{R} \times\{0,1\}$. Applying Lemma 1.5 (the Bellman principle) of [13] to the value function defined above, we have

$$
v(x, y, z)=\sup _{(\kappa ., \ell,, c .) \in \mathcal{A}(\mathbb{G})} \mathrm{E}_{x, y, z}\left[\int_{0}^{\zeta_{t}} \mathrm{e}^{-\alpha s} U\left(c_{s} x_{s}\right) \mathrm{d} s+\mathrm{e}^{-\alpha \zeta_{t}} v\left(x_{\zeta_{t}}, y_{\zeta_{t}}, z_{\zeta_{t}}\right)\right],
$$

for all $\mathbb{G}$-stopping times $\zeta_{t}:=\zeta \wedge t, t \geq 0$. Hence, the HJB equation associated with $v(x, y, z)$ is given by

$$
\begin{aligned}
\alpha v(x, y, z)= & r x v_{x}(x, y, z) \\
+\sup _{(\kappa, \ell, c) \in A_{1} \times A_{2} \times A_{3}}[ & U(c x)+(b-r) \ell x v_{x}(x, y, z)-c x v_{x}(x, y, z) \\
& +\frac{1}{2} a^{2} \ell^{2} x^{2} v_{x x}(x, y, z) \\
& +(1-z) \lambda(y)\left(\frac{\rho}{\eta(y)}\right) \kappa x v_{x}(x, y, z) \\
& +(v(x-x \kappa \rho, y, 1)-v(x, y, 0))(1-z) \lambda(y)] \\
+\mu(y) v_{y}(x, y, z)+ & \frac{1}{2} v_{y y}(x, y, z)
\end{aligned}
$$

for $(x, y, z) \in(0, \infty) \times \mathbb{R} \times\{0,1\}$, where $v_{x}:=\partial v / \partial x, v_{y}:=\partial v / \partial y, v_{x x}:=\partial v^{2} / \partial x^{2}$, and $v_{y y}:=\partial v^{2} / \partial y^{2}$.

Since $z=0$ or 1 , we consider two cases:

$$
\widetilde{v}(x, y):=v(x, y, 0) \quad \text { (the pre-default case) }
$$

and

$$
\bar{v}(x, y):=v(x, y, 1) \quad(\text { the post-default case })
$$


Since the post-default case has been well studied, we only provide results for which the post-default value function is given by

$$
\bar{v}(x, y)=\frac{1}{\alpha} \log x+R_{\alpha},
$$

where

$$
R_{\alpha}:=\frac{1}{\alpha^{2}}\left(\frac{(b-r)^{2}}{2 a^{2}}+r\right)+\frac{\log \alpha-1}{\alpha}, \quad y \in \mathbb{R},
$$

and the optimal control strategies are given by

$$
\bar{\ell}^{*}=\frac{b-r}{a^{2}}, \quad \bar{\kappa}^{*}=0, \quad \bar{c}^{*}=\alpha .
$$

In the following, we will concentrate on the pre-default case. According to (3.3), $\widetilde{v}$ obeys the following dynamics:

$$
\begin{aligned}
\alpha \widetilde{v}= & r x \widetilde{v}_{x}+\mu(y) \widetilde{v}_{y}+\frac{1}{2} \widetilde{v}_{y y} \\
& +\sup _{(\ell, c) \in A_{2} \times A_{3}}\left[U(c x)+(b-r) \ell x \widetilde{v}_{x}-c x \widetilde{v}_{x}+\frac{1}{2} a^{2} \ell^{2} x^{2} \widetilde{v}_{x x}\right] \\
& +\sup _{\kappa \in A_{1}}\left[\lambda(y)\left(\frac{\rho}{\eta(y)}\right) \kappa x \widetilde{v}_{x}+(\bar{v}(x-x \kappa \rho, y)-\widetilde{v}(x, y)) \lambda(y)\right] .
\end{aligned}
$$

It is not hard to verify that $\tilde{v}(x, y)$ admits the form

$$
\widetilde{v}(x, y)=\frac{1}{\alpha} \log x+\widetilde{\omega}(y), \quad(x, y) \in(0, \infty) \times \mathbb{R} .
$$

Substituting this into (3.7), it follows that $\widetilde{\omega}(y)$ is governed by

$$
\begin{aligned}
& \frac{1}{2} \widetilde{\omega}_{y y}+\mu(y) \widetilde{\omega}_{y}-(\alpha+\lambda(y)) \widetilde{\omega}+\log \alpha-1+\lambda(y) R_{\alpha} \\
& +\frac{1}{\alpha}\left(r+\frac{(b-r)^{2}}{2 a^{2}}+\frac{\lambda(y)(1-\eta(y))}{\eta(y)}+\lambda(y) \log (\eta(y))\right)=0,
\end{aligned}
$$

and the maximum points in (3.7) are given by

$$
\tilde{\ell}^{*}=\frac{b-r}{a^{2}}, \quad \widetilde{\kappa}^{*}=\frac{1-\eta(y)}{\rho}, \quad \widetilde{c}^{*}=\alpha .
$$

Actually, in the next section we will prove that $\left(\widetilde{\ell}^{*}, \widetilde{\kappa}^{*}, \widetilde{c}^{*}\right)$ is the optimal control strategy.

Next we adopt the so called sub/super-solution method to obtain the existence and uniqueness of the solution to Equation (3.8). (An advantage of the sub/super-solution method is that we can obtain some proper bounds for the unique classical solution to (3.8). Since the linear equation (3.8) has variable coefficients, the 'fundamental set of solutions' approach seems to be unavailable; see also [22].) Rewrite (3.8) as

$$
\frac{1}{2} \widetilde{\omega}_{y y}+\mu(y) \widetilde{\omega}_{y}-(\alpha+\lambda(y)) \widetilde{\omega}+\alpha R_{\alpha}+\frac{\lambda(y)}{\alpha} h(\eta(y))=0,
$$

where

$$
h(x):=\frac{1-x}{x}+\log x+\alpha R_{\alpha}, \quad x \in(0,1] .
$$


Define

$$
\begin{gathered}
L w=-\frac{1}{2} w_{y y}-\mu(y) w_{y}, \\
f(y, w)=-(\alpha+\lambda(y)) w+\alpha R_{\alpha}+\frac{\lambda(y)}{\alpha} h(\eta(y)) .
\end{gathered}
$$

Recall that $\underline{w}(y)$ is said to be a sub-solution of (3.9) on the real line if $L \underline{w} \leq f(y, \underline{w})$, and that $\bar{w}(y)$ is said to be a super-solution of (3.9) on the real line if $L \bar{w} \geq \bar{f}(y, \bar{w})$ ). Moreover, if $\underline{w}(y) \leq \bar{w}(y)$ for all $y \in \mathbb{R}$ then $(\underline{w}, \bar{w})$ is called a sub/super-solution ordered pair of (3.9) (see Definition 3.1 of [11]). Now we have the following result.

Proposition 3.1. Let the default intensity function $\lambda(y) \geq 2 \bar{C}-\alpha$ for all $y \in \mathbb{R}$. Then, under assumptions (H1)-(H3), (3.9) admits a classical solution $\widetilde{\omega}(y)$ such that

$$
R_{\alpha} \leq \widetilde{\omega}(y) \leq \overline{\widetilde{\omega}}(y):=C_{1} y^{2}+C_{2} \text { for all } y \in \mathbb{R}
$$

for some $C_{1}>0$ and $C_{2}>R_{\alpha}$, where $R_{\alpha}$ is defined in (3.5).

Proof. We use the so-called sub/super-solution of partial differential equations (see, e.g. [11]). Note that, for all $x \in(0,1]$, it holds that

$$
0 \leq x^{-1}(1-x)+\log x \leq x^{-1} .
$$

As a consequence, the mapping $x \rightarrow h(x)$ defined by (3.10) satisfies

$$
\alpha R_{\alpha} \leq h(x)<\alpha R_{\alpha}+x^{-1} \text { for all } x \in(0,1],
$$

so we have

$$
\frac{\alpha R_{\alpha}+(\lambda(y) / \alpha) h(\eta(y))}{\alpha+\lambda(y)} \geq \frac{\alpha R_{\alpha}+(\lambda(y) / \alpha) \alpha R_{\alpha}}{\alpha+\lambda(y)}=R_{\alpha} .
$$

Since $R_{\alpha}$ is a constant, $L R_{\alpha}=0$. Then, for each constant $C \leq R_{\alpha}$, it is a sub-solution of (3.9).

On the other hand, for $y \in \mathbb{R}$ fixed, there exists some $\theta \in[0, y]$ such that

$$
\begin{aligned}
L \overline{\widetilde{\omega}}(y) & =-C_{1}-2 C_{1} y \mu(y) \\
& =-C_{1}-2 C_{1} y[\mu(y)-\mu(0)+\mu(0)] \\
& =-C_{1}-2 C_{1} y\left[y \mu_{y}(\theta)+\mu(0)\right] \\
& \geq-2 C_{1} \bar{C} y^{2}-2 C_{1} \mu(0) y-C_{1} .
\end{aligned}
$$

It follows from (3.11) that

$$
\begin{aligned}
f(y, \widetilde{\omega}(y)) & =-(\alpha+\lambda(y))\left[C_{1} y^{2}+C_{2}\right]+\alpha R_{\alpha}+\frac{\lambda(y)}{\alpha} h(\eta(y)) \\
& \leq-(\alpha+\lambda(y)) C_{1} y^{2}+\frac{\lambda(y)}{\alpha \eta_{m}}+(\alpha+\lambda(y))\left(R_{\alpha}-C_{2}\right) .
\end{aligned}
$$

Taking $C_{2}>R_{\alpha}$ large enough (since $\lambda(\cdot)$ is nonnegative), it holds that

$$
L \overline{\widetilde{\omega}}(y) \geq f(y, \overline{\widetilde{\omega}}(y)) \quad \text { for all } y \in \mathbb{R} .
$$

This shows that $\widetilde{\widetilde{\omega}}(y)$ is a super-solution of (3.9). Hence, $\left(R_{\alpha}, \widetilde{\widetilde{\omega}}(y)\right)$ is a sub/super-solution ordered pair of (3.9). Now define

$$
\bar{H}(y, p, q):=-\mu(y) q+(\alpha+\lambda(y)) p-\alpha R_{\alpha}-\frac{\lambda(y)}{\alpha} h(\eta(y)) .
$$


It is not hard to conclude that $p \rightarrow \bar{H}(y, p, q)$ is strictly increasing. On the other hand, let $\triangle=[y, \bar{y}]$ be an arbitrary finite interval on the real line, and let $\Phi=\max \left\{\sup _{y \in \Delta}|\widetilde{\widetilde{\omega}}(y)|,\left|R_{\alpha}\right|\right\}$. Then it follows from assumptions (H1) and (H2) that there exists $\theta \geq 0$ such that, for all $y \in \triangle$ and $|p| \leq 3 \Phi$,

$$
\begin{aligned}
|\bar{H}(y, p, q)| \leq & \left(\left|\mu_{y}(\theta)\right||y|+|\mu(0)|\right)|q|+\left(\alpha+C+C|y|^{\delta}\right)\left(|p|+R_{\alpha}\right) \\
& +\frac{1}{\alpha}\left(C+C|y|^{\delta}\right) \eta_{m}^{-1} \\
\leq & (\bar{C}|y|+|\mu(0)|)|q|+\left(\alpha+C+C|y|^{\delta}\right)\left(|p|+\alpha R_{\alpha}\right) \\
& +\frac{1}{\alpha}\left(C+C|y|^{\delta}\right) \eta_{m}^{-1} \\
\leq & \frac{1}{2} q^{2}+\frac{1}{2}(\bar{C}|y|+|\mu(0)|)^{2}+\left(\alpha+C+C|y|^{\delta}\right)\left(|p|+\alpha R_{\alpha}\right) \\
& +\frac{1}{\alpha}\left(C+C|y|^{\delta}\right) \eta_{m}^{-1} \\
\leq & \frac{1}{2} q^{2}+\Psi,
\end{aligned}
$$

where

$$
\Psi=\frac{1}{2}\left(\bar{C} y_{m}+|\mu(0)|\right)^{2}+\left(\alpha+C+C y_{m}^{\delta}\right)\left(3 \Phi+\alpha R_{\alpha}\right)+\frac{1}{\alpha}\left(C+C y_{m}^{\delta}\right) \eta_{m}^{-1}
$$

with $y_{m}:=\max \{|\underline{y}|,|\bar{y}|\}$. Thus, the proposition follows from Lemma 3.9 and Theorem 3.8 of [11].

\section{Verification theorem}

In this section we prove a verification theorem, in which we verify that the pre-default value function is $\widetilde{v}(x, y)=(1 / \alpha) \log x+\widetilde{\omega}(y)$, where $\widetilde{\omega}(y)$ is given in Proposition 3.1 , and that the optimal control strategy is given by

$$
\kappa_{t}^{*}=\kappa^{*}\left(y_{t}\right)=\frac{1-\eta\left(y_{t}\right)}{\rho}, \quad \ell_{t}^{*}=\frac{b-r}{a^{2}}, \quad c_{t}^{*}=\alpha .
$$

Firstly, we have the following lemma.

Lemma 4.1. Suppose that assumptions (H1)-(H3) hold and that $\alpha>\delta \bar{C}$. Then the triplet $\left(\kappa_{t}^{*}, \ell_{t}^{*}, c_{t}^{*}\right)_{t \geq 0}$ given in (4.1) is an admissible control, i.e. $\left(\kappa_{t}^{*}, \ell_{t}^{*}, c_{t}^{*}\right)_{t \geq 0} \in \mathcal{A}(\mathbb{G})$. Moreover, the following properties hold:

(a) $\lim _{T \rightarrow \infty} \mathrm{e}^{-\alpha T} \mathrm{E}\left[\int_{0}^{T} \ell_{s}^{2} \mathrm{~d} s\right]=0$,

(b) $\lim _{T \rightarrow \infty} \mathrm{e}^{-\alpha T} \mathrm{E}\left[\int_{0}^{T \wedge \tau} \lambda\left(y_{s}\right) \log ^{2}\left(1-\rho \kappa_{s}\right) \mathrm{d} s\right]=0$,

(c) $c_{t} \leq N$ for some $N>0$, P-almost surely (P-a.s.).

Proof. Obviously, the $\left(\ell_{t}^{*}\right)_{t \geq 0}$ satisfy (a). Note that, for $y \in \mathbb{R}, \eta(y) \in(0,1]$, and so $\kappa_{t}^{*} \in[0,1 / \rho)$ for each $t \geq 0$. Also, $c_{t}^{*}$ obviously satisfies (c). On the other hand, it follows from assumption (H3) that $1 \leq \eta^{-1}(y) \leq \eta_{m}^{-1}$ for all $y \in \mathbb{R}$. Thus, in light of assumption (H2) 
and Lemma 2.1,

$$
\begin{aligned}
0 & \leq \mathrm{e}^{-\alpha T} \mathrm{E}\left[\int_{0}^{T \wedge \tau} \lambda\left(y_{t}\right) \log ^{2}\left(1-\rho \kappa_{t}^{*}\right) \mathrm{d} t\right] \\
& =\mathrm{e}^{-\alpha T} \mathrm{E}\left[\int_{0}^{T \wedge \tau} \lambda\left(y_{t}\right) \log ^{2}\left(\frac{1}{\eta\left(y_{t}\right)}\right) \mathrm{d} t\right] \\
& \leq C \log ^{2}\left(\eta_{m}\right) \mathrm{e}^{-\alpha T} \mathrm{E}\left[\int_{0}^{T}\left(1+\left|y_{t}\right|^{\delta}\right) \mathrm{d} t\right] \\
& \rightarrow 0
\end{aligned}
$$

as $T \rightarrow \infty$. This proves that $\left(\kappa_{t}^{*}\right)_{t \geq 0}$ satisfies (b).

Now we are in a position to state the main result of the paper.

Theorem 4.1. (Verification theorem.) Suppose that assumptions (H1)-(H3) hold. Assume that $\alpha>2 \max \{\delta, 2\} \bar{C}$ (where $\bar{C}$ is given in (H1) and $\delta \geq 1$ is given in (H2)). Define a function on $(0, \infty) \times \mathbb{R} \times\{0,1\}$ by

$$
\hat{v}(x, y, z)=\frac{1}{\alpha} \log x+z R_{\alpha}+(1-z) \widetilde{\omega}(y),
$$

where $R_{\alpha}$ is presented in (3.5) and $\widetilde{\omega}(y)$ is a classical solution to (3.9).

(a) For all admissible control policies $\left(\kappa_{t}, \ell_{t}, c_{t}\right)_{t \geq 0} \in \mathcal{A}(\mathbb{G})$, it holds that

$$
\hat{v}(x, y, z) \geq \mathrm{E}_{x, y, z}\left[\int_{0}^{\infty} \mathrm{e}^{-\alpha t} U\left(c_{t} x_{t}\right) \mathrm{d} t\right],
$$

with $(x, y, z) \in(0, \infty) \times \mathbb{R} \times\{0,1\}$.

(b) Define

$$
\begin{gathered}
\ell_{t}^{*}=\frac{b-r}{a^{2}}, \quad t \geq 0, \\
c_{t}^{*}=\alpha, \quad t \geq 0,
\end{gathered}
$$

and

$$
\kappa_{t}^{*}=\kappa^{*}\left(y_{t}\right)= \begin{cases}\frac{1-\eta\left(y_{t}\right)}{\rho}, & 0 \leq t<\tau, \\ 0, & t \geq \tau\end{cases}
$$

Then $\left(\kappa_{t}^{*}, \ell_{t}^{*}, c_{t}^{*}\right)_{t \geq 0} \in \mathcal{A}(\mathbb{G})$ and its value function $v=\hat{v}$, i.e. for $(x, y, z) \in(0, \infty) \times$ $\mathbb{R} \times\{0,1\}$,

$$
v(x, y, z):=\mathrm{E}_{x, y, z}\left[\int_{0}^{\infty} \mathrm{e}^{-\alpha t} U\left(c_{t}^{*} x_{t}^{*}\right) \mathrm{d} t\right]=\hat{v}(x, y, z),
$$

where $\left(x_{t}^{*}\right)_{t \geq 0}$ is the wealth process satisfying $(3.1)$ with $\left(\kappa_{t}, \ell_{t}, c_{t}\right)_{t \geq 0}$ replaced by $\left(\kappa_{t}^{*}, \ell_{t}^{*}, c_{t}^{*}\right)_{t \geq 0}$. 
Proof. Recall $x_{t}, y_{t}$, and $z_{t}$ in Sections 2 and 3. Let $\hat{v}$ be as defined in (4.2). Then the Itô formula yields

$$
\begin{aligned}
\mathrm{d} \hat{v}\left(x_{t}, y_{t}, z_{t}\right)= & x_{t} \hat{v}_{x}\left[r-c_{t}+\ell_{t}(b-r)+\kappa_{t}\left(1-z_{t}\right) \lambda\left(y_{t}\right)\left(\frac{\rho}{\eta\left(y_{t}\right)}\right)\right] \mathrm{d} t \\
+ & {\left[\frac{1}{2} x_{t}^{2} \hat{v}_{x x} \ell_{t}^{2} a^{2}+\frac{1}{2} \hat{v}_{y y}+\mu\left(y_{t}\right) \hat{v}_{y}\right] \mathrm{d} t } \\
+ & {\left[\hat{v}\left(x_{t}-x_{t} \kappa_{t}\left(1-z_{t}\right) \rho, y_{t}, z_{t}+1\right)-\hat{v}\left(x_{t}, y_{t}, z_{t}\right)\right] } \\
& \times\left(1-z_{t}\right) \lambda\left(y_{t}\right) \mathrm{d} t+\mathrm{d} M_{t}, \quad t \geq 0,
\end{aligned}
$$

where

$$
\begin{aligned}
M_{t}= & \int_{0}^{t} x_{s} \hat{v}_{x}\left(x_{s}, y_{s}, z_{s}\right) \ell_{s} a \mathrm{~d} W_{s}+\int_{0}^{t} a \hat{v}_{y}\left(x_{s}, y_{s}, z_{s}\right) \mathrm{d} \breve{W}_{s} \\
& +\int_{0}^{t+}\left[\hat{v}\left(x_{s-}-x_{s-} \kappa_{s-}\left(1-z_{s-}\right) \rho, y_{s}, z_{s-}+1\right)-\hat{v}\left(x_{s-}, y_{s}, z_{s-}\right)\right] \mathrm{d} m_{s} .
\end{aligned}
$$

By the assumptions in Section $2,\left(M_{t}\right)_{t \geq 0}$ is a $(\mathrm{P}, \mathbb{G})$-adapted càdlàg martingale. Note that

$$
x \hat{v}_{x}=\alpha^{-1}, \quad x^{2} \hat{v}_{x x}=-\alpha^{-1} .
$$

Consequently, by some standard calculus and (3.8), we have

$$
\begin{aligned}
\mathrm{d} \hat{v}\left(x_{t}, y_{t}, z_{t}\right) & \leq \alpha\left[z_{t} R_{\alpha}+\left(1-z_{t}\right) \widetilde{\omega}\left(y_{t}\right)\right] \mathrm{d} t+\mathrm{d} M_{t} \\
& =\left[\alpha \hat{v}\left(x_{t}, y_{t}, z_{t}\right)-U\left(c_{t} x_{t}\right)\right] \mathrm{d} t+\mathrm{d} M_{t},
\end{aligned}
$$

where $\left(\kappa_{t}^{*}, \ell_{t}^{*}, c_{t}^{*}\right)_{t \geq 0}$ is given in (4.1). So

$$
\begin{aligned}
\mathrm{d}\left[\mathrm{e}^{-\alpha t} \hat{v}\left(x_{t}, y_{t}, z_{t}\right)\right] & =\mathrm{e}^{-\alpha t} \mathrm{~d} \hat{v}\left(x_{t}, y_{t}, z_{t}\right)-\alpha \mathrm{e}^{-\alpha t} \hat{v}\left(x_{t}, y_{t}, z_{t}\right) \mathrm{d} t \\
& \leq \mathrm{e}^{-\alpha t}\left[\alpha \hat{v}\left(x_{t}, y_{t}, z_{t}\right)-U\left(c_{t} x_{t}\right)\right] \mathrm{d} t+\mathrm{e}^{-\alpha t} \mathrm{~d} M_{t}-\alpha \mathrm{e}^{-\alpha t} \hat{v}\left(x_{t}, y_{t}, z_{t}\right) \mathrm{d} t .
\end{aligned}
$$

This implies that, for $T>0$,

$$
\hat{v}(x, y, z) \geq \mathrm{E}_{x, y, z}\left[\int_{0}^{T} \mathrm{e}^{-\alpha s} U\left(c_{s} x_{s}\right) \mathrm{d} s\right]+\mathrm{E}_{x, y, z}\left[\mathrm{e}^{-\alpha T} \hat{v}\left(x_{T}, y_{T}, z_{T}\right)\right] .
$$

On the other hand, from Itô's formula (see, e.g. [24, p. 78]), it follows that

$$
\begin{aligned}
\log x_{T}= & \log x_{0}+\int_{0}^{T} \ell_{s} a \mathrm{~d} W_{s}+\int_{0}^{T} \log \left(1-\kappa_{s-} \rho\left(1-z_{s-}\right)\right) \mathrm{d} m_{s} \\
& +\int_{0}^{T}\left[r-c_{s}+\ell_{s}(b-r)+\kappa_{s}\left(1-z_{s}\right) \lambda\left(y_{s}\right)\left(\frac{\rho}{\eta\left(y_{s}\right)}\right)-\frac{1}{2} \ell_{s}^{2} a^{2}\right] \mathrm{d} s \\
& +\int_{0}^{T \wedge \tau} \lambda\left(y_{s}\right) \log \left(1-\kappa_{s} \rho\left(1-z_{s}\right)\right) \mathrm{d} s .
\end{aligned}
$$

Once again, since $\left(\kappa_{t}, \ell_{t}, c_{t}\right)_{t \geq 0} \in \mathcal{A}(\mathbb{G})$, we have, for $x>0$,

$$
\limsup _{T \rightarrow \infty} \mathrm{e}^{-\alpha T} \mathrm{E}_{x}\left[\int_{0}^{T}-\frac{1}{2} a^{2} \ell_{s}^{2} \mathrm{~d} s\right] \geq-\frac{1}{2} a^{2} \lim _{T \rightarrow \infty} \mathrm{e}^{-\alpha T} \mathrm{E}_{x}\left[\int_{0}^{T} \ell_{s}^{2} \mathrm{~d} s\right]=0 .
$$


It follows that

$$
\begin{aligned}
& \limsup _{T \rightarrow \infty} \mathrm{e}^{-\alpha T} \mathrm{E}_{x}\left[\int_{0}^{T}\left(r+\ell_{s}(b-r)\right) \mathrm{d} s\right] \\
& \geq \lim _{T \rightarrow \infty}\left[r T-\frac{1}{2}(b-r)^{2} T\right] \mathrm{e}^{-\alpha T}-\frac{1}{2} \lim _{T \rightarrow \infty} \mathrm{e}^{-\alpha T} \mathrm{E}_{x}\left[\int_{0}^{T} \ell_{s}^{2} \mathrm{~d} s\right] \\
& =0 \text {. }
\end{aligned}
$$

Moreover, it holds that

$$
\limsup _{T \rightarrow \infty} \mathrm{e}^{-\alpha T} \mathrm{E}_{x}\left[\int_{0}^{T}-c_{s} \mathrm{~d} s\right] \geq-N \lim _{T \rightarrow \infty} \mathrm{e}^{-\alpha T} T=0 .
$$

On the other hand, by virtue of Lemma 2.1,

$$
\begin{aligned}
& \limsup _{T \rightarrow \infty} \mathrm{e}^{-\alpha T} \mathrm{E}_{x}\left[\int_{0}^{T} \kappa_{s}\left(1-z_{s}\right) \lambda\left(y_{s}\right) \frac{\rho}{\eta\left(y_{s}\right)} \mathrm{d} s\right] \\
& \geq \liminf _{T \rightarrow \infty} \mathrm{e}^{-\alpha T} \mathrm{E}_{x}\left[-\int_{0}^{T \wedge \tau} \kappa_{s} \frac{\rho}{\eta\left(y_{s}\right)} \lambda\left(y_{s}\right) \mathrm{d} s\right] \\
& \geq \frac{1}{2} \liminf _{T \rightarrow \infty} \mathrm{e}^{-\alpha T} \mathrm{E}_{x}\left[-\int_{0}^{T \wedge \tau} \kappa_{s}^{2} \lambda\left(y_{s}\right) \mathrm{d} s\right] \\
&+\frac{1}{2} \liminf _{T \rightarrow \infty} \mathrm{e}^{-\alpha T} \mathrm{E}_{x}\left[-\int_{0}^{T \wedge \tau}\left(\frac{\rho}{\eta\left(y_{s}\right)}\right)^{2} \lambda\left(y_{s}\right) \mathrm{d} s\right] \\
& \geq \frac{1}{2}\left[\frac{1}{\rho^{2}}+\frac{\rho^{2}}{\eta_{m}^{2}}\right] \lim _{T \rightarrow \infty} \mathrm{e}^{-\alpha T} \mathrm{E}_{x}\left[-C T-C \int_{0}^{T}\left|y_{s}\right|^{\delta} \mathrm{d} s\right] \\
&= 0
\end{aligned}
$$

and

$$
\begin{aligned}
& \underset{T \rightarrow \infty}{\limsup _{T \rightarrow \infty}} \mathrm{e}^{-\alpha T} \mathrm{E}_{x}\left[\int_{0}^{T} \lambda\left(y_{s}\right)\left(1-z_{s}\right) \log \left(1-\kappa_{s} \rho\left(1-z_{s}\right)\right) \mathrm{d} s\right] \\
& \geq \frac{1}{2} \lim _{T \rightarrow \infty} \mathrm{e}^{-\alpha T} \mathrm{E}_{x}\left[-\int_{0}^{T \wedge \tau} \lambda\left(y_{s}\right) \log ^{2}\left(1-\kappa_{s} \rho\right) \mathrm{d} s\right] \\
&+\frac{1}{2} \lim _{T \rightarrow \infty} \mathrm{e}^{-\alpha T} \mathrm{E}_{x}\left[-\int_{0}^{T}\left(C+C\left|y_{s}\right|^{\delta}\right) \mathrm{d} s\right] \\
&= 0 .
\end{aligned}
$$

Based on the above derivations, part (a) follows from Proposition 3.1 and (4.9). Recall (3.8). Then a similar argument as that used for (4.9) shows that

$$
\hat{v}(x, y, z)=\mathrm{E}_{x, y, z}\left[\int_{0}^{T} \mathrm{e}^{-\alpha s} \log \left(c_{s}^{*} x_{s}^{*}\right) \mathrm{d} s\right]+\mathrm{e}^{-\alpha T} \mathrm{E}_{x, y, z}\left[\hat{v}\left(x_{T}^{*}, y_{T}, z_{T}\right)\right] .
$$


Combining the survival probability (2.3) and Proposition 3.1, we have

$$
\begin{aligned}
\mathrm{E}_{x, y, z} & {\left[\hat{v}\left(x_{T}^{*}, y_{T}, z_{T}\right)\right] } \\
& =\frac{1}{\alpha} \mathrm{E}\left[\log x_{T}^{*}\right]+R_{\alpha} \mathrm{P}(\tau \leq T)+\mathrm{E}_{x, y, z}\left[\left(1-z_{T}\right) \tilde{\omega}\left(y_{T}\right)\right] \\
& \leq \frac{1}{\alpha} \mathrm{E}\left[\log x_{T}^{*}\right]+R_{\alpha}+\mathrm{E}_{x, y, z}\left[C_{1} y_{T}^{2}+C_{2}\right],
\end{aligned}
$$

where the constants $C_{1}$ and $C_{2}$ are given in Proposition 3.1. Similarly as in the proof of part (a), using Lemma 2.1,

$$
\liminf _{T \rightarrow \infty} \mathrm{e}^{-\alpha T} \mathrm{E}_{x, y, z}\left[\hat{v}\left(x_{T}^{*}, y_{T}, z_{T}\right)\right] \leq 0 .
$$

Thus, apply (a), the Fatou lemma, and (4.10)-(4.11) to conclude that (b) holds. This completes the proof.

\section{Appendix A. Price dynamics for a defaultable bond}

In this appendix we derive the price dynamics for a perpetual defaultable bond that pays a constant coupon $\widetilde{C}$ per unit time. Here the spot interest rate $r$ and the loss rate, given default $\rho \in(0,1)$, are two positive constants.

Let $\mathrm{Q}$ be the risk-neutral probability measure. Suppose that the P-default intensity $\lambda$ and the default risk premium $1 / \eta$ are two positive constants. Then the $\mathrm{Q}$-default intensity is $\lambda^{\mathrm{Q}}=\lambda / \eta$.

Under the recovery of market value scheme, the pre-default value of the bond at time $t$ is given by

$$
V_{t}=\mathrm{E}^{\mathrm{Q}}\left[\int_{t}^{\infty} \widetilde{C} \mathrm{e}^{-r(s-t)} \mathbf{1}_{\{\tau>s\}} \mathrm{d} s \mid g_{t}\right]+\mathrm{E}^{\mathrm{Q}}\left[\mathrm{e}^{-r(\tau-t)}(1-\rho) V_{\tau-} \mid g_{t}\right] \mathbf{1}_{\{\tau>t\}} .
$$

From Lemma 5.1.2 of [5] we have

$$
V_{t}=\frac{\widetilde{C}}{r+\lambda \mathrm{Q}}+(1-\rho) \lambda^{\mathrm{Q}} \mathrm{E}^{\mathrm{Q}}\left[\int_{t}^{\infty} \mathrm{e}^{-\left(r+\lambda^{\mathrm{Q}}\right)(s-t)} V_{s} \mathrm{~d} s \mid \mathcal{F}_{t}\right] \quad \text { on }\{\tau>t\} .
$$

Differentiating (A.2) with respect to $t$ yields

$$
\begin{aligned}
\mathrm{d} V_{t} & =-(1-\rho) \lambda^{\mathrm{Q}} V_{t} \mathrm{~d} t+\left(r+\lambda^{\mathrm{Q}}\right)\left(V_{t}-\frac{\widetilde{C}}{r+\lambda \mathrm{Q}}\right) \mathrm{d} t \\
& =\widetilde{r} V_{t} \mathrm{~d} t-\widetilde{C} \mathrm{~d} t \quad \text { on }\{\tau>t\},
\end{aligned}
$$

with the adjusted interest rate $\tilde{r}=r+\rho \lambda \mathrm{Q}$. By some standard calculus,

$$
V_{t}=\frac{\widetilde{C}}{\widetilde{r}}+\left(V_{0}-\frac{\widetilde{C}}{\widetilde{r}}\right) \mathrm{e}^{\tilde{r} t}
$$

We now define the price process for a perpetual defaultable bond under $\mathrm{Q}$ as follows (see the price process (1) in [4]):

$$
p_{t}=\mathbf{1}_{\{\tau>t\}} V_{t}+\mathbf{1}_{\{\tau \leq t\}}(1-\rho) V_{\tau} \mathrm{e}^{r(t-\tau)} .
$$

Recall that $z_{t}=\mathbf{1}_{\{\tau \leq t\}}$, and note that $\mathrm{d} z_{t}=\left(1-z_{t}\right) \mathrm{d} z_{t}, V_{t} \mathrm{~d} z_{t}=V_{\tau} \mathrm{d} z_{t}$, and $\mathrm{e}^{r(t-\tau)} \mathrm{d} z_{t}=\mathrm{d} z_{t}$. Applying Itô's formula to (A.5), we obtain

$$
\mathrm{d} p_{t}=r p_{t} \mathrm{~d} t+\rho \lambda^{\mathrm{Q}}\left(1-z_{t}\right) p_{t} \mathrm{~d} t-\left(1-z_{t}\right) \widetilde{C} \mathrm{~d} t-\rho p_{t} \mathrm{~d} z_{t} .
$$


Moreover, the price dynamics of $\left(p_{t}\right)_{t \geq 0}$ under the physical probability measure $\mathrm{P}$ is

$$
\mathrm{d} p_{t}=r p_{t} \mathrm{~d} t+\rho \lambda\left(\frac{1}{\eta}-1\right)\left(1-z_{t}\right) p_{t} \mathrm{~d} t-\left(1-z_{t}\right) \widetilde{C} \mathrm{~d} t-\rho p_{t} \mathrm{~d} m_{t},
$$

where $m_{t}=z_{t}-\int_{0}^{t}\left(1-z_{s}\right) \lambda \mathrm{d} s$ is a P-martingale.

\section{Appendix B. Numerical results}

In this appendix we present a parametric sensitivity analysis for the optimal control $\left(\kappa_{t}^{*}\right)_{t \geq 0}$ of the defaultable bond and the value function $v$ obtained in (4.2) using some numerical simulations.

For ease of exposition, we adopt the following abbreviations.

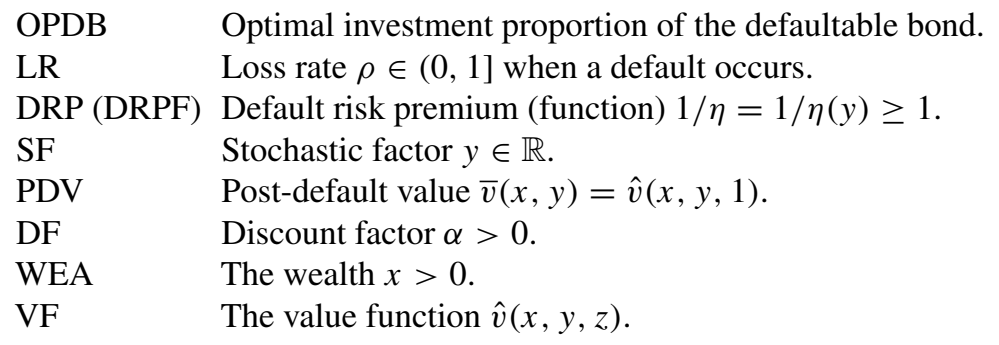

Suppose that $\lambda(y)=\varepsilon+|y|(\varepsilon>0), \mu(y)=\bar{C}(1-y)$, and that the DRPF is a constant, i.e. $\eta(y) \equiv \eta \in(0,1]$. We quote some parameters from [4]:

\begin{tabular}{cccccccc}
$\rho$ & $\alpha$ & $r$ & $a$ & $\bar{C}$ & $\eta$ & $b$ & $\varepsilon$ \\
\hline $0.52,0.62,0.9$ & $2 \bar{C}+\varepsilon$ & 0 & 0.15 & 0.5 & 0.395257 & 0.06765 & 0.01
\end{tabular}

We begin by discussing the parametric sensitivity in the optimal control $\kappa^{*}$ for the defaultable bond. Recall (4.5). The optimal control $\kappa^{*}$ is completely characterized by the LR and DRP, and it is independent of the risk-free interest rate $r$ and of the default intensity process $\lambda_{t}$ (different from that in [14] for the exponential utility). From Figures 1 and 2, we find that, for a fixed DRP, the OPDB increases while the LR decreases; for a fixed LR, the investor increases the amount of OPDB when the DRP increases (a similar phenomena appears in [14]). However, if the DRPF relies on some SF (not constant) then the OPDB is a joint function of the LR and SF variables. Figure 3 displays the relationship between the OPDB, LR, and SF for a DRPF. (The choice of the DRPF in Figure 3 is not experiential, we use it only to illuminate the analysis.)

We now investigate the parametric sensitivity of the value function $\hat{v}(x, y, z)$ given by (4.2). Owing to the form of the equation,

$$
\frac{1}{2} \widetilde{\omega}_{y y}+\bar{C}(1-y) \widetilde{\omega}_{y}-(\alpha+\varepsilon+|y|) \widetilde{\omega}+\alpha R_{\alpha}+\frac{\varepsilon+|y|}{\alpha} h(\eta)=0,
$$

with $y \in \mathbb{R}$, we do not expect (B.1) to admit a closed-form solution. 


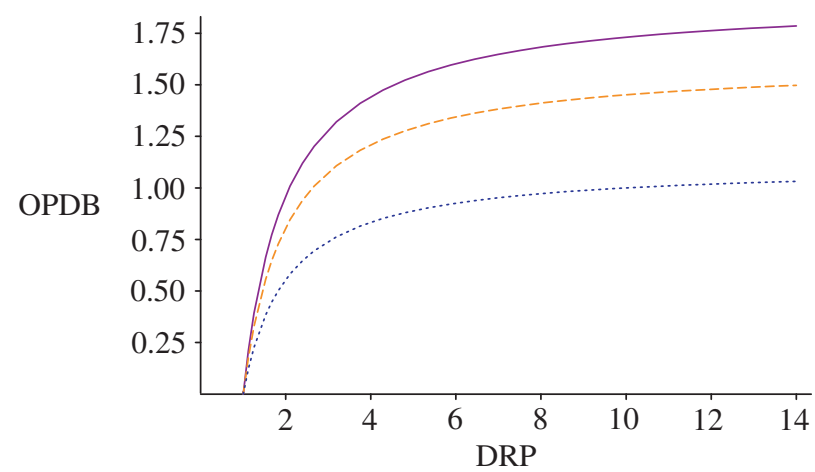

FIGURE 1: The OPDB versus the DRP for $\mathrm{LR}=0.52,0.62,0.9$. The solid, dashed, and dotted lines correspond to $R=0.52,0.6$, and 0.9 , respectively.

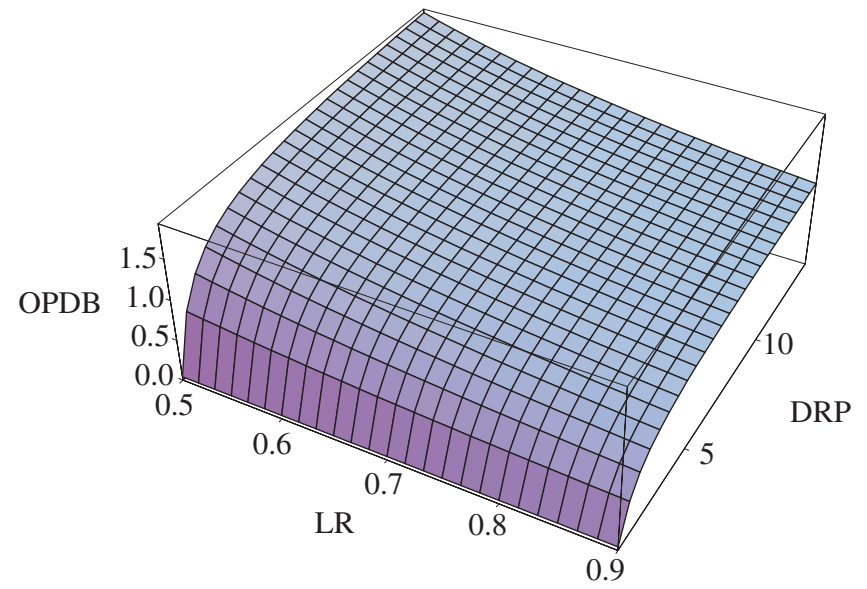

FIGURE 2: The OPDB versus the LR and DRP.

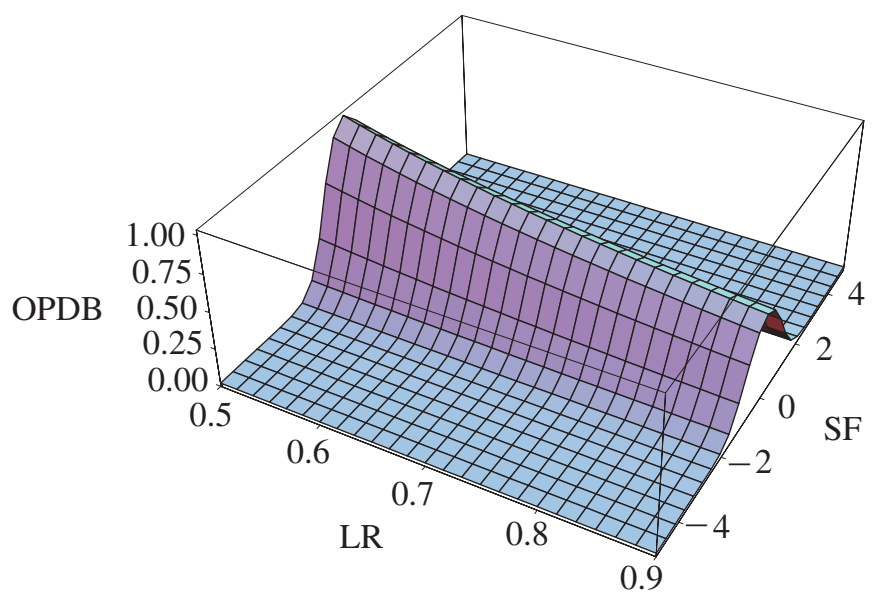

Figure 3: The OPDB versus the LR and SF with $\eta_{m}=\frac{1}{2}$ and DRPF $\eta^{-1}(y)=1+\mathrm{e}^{-y^{2}}$. 
Thanks to Proposition 3.1, we can obtain the lower and upper bounds:

$$
\begin{aligned}
\hat{v}(x, y, z) & =\frac{1}{\alpha} \log x+z R_{\alpha}+(1-z) \widetilde{\omega}(y) \\
& \geq \frac{1}{\alpha} \log x+z R_{\alpha}+(1-z) R_{\alpha} \\
& =\frac{1}{\alpha} \log x+R_{\alpha} \\
& =\bar{v}(x, y), \\
\hat{v}(x, y, z) & \leq \frac{1}{\alpha} \log x+R_{\alpha}+C_{2}+C_{1} y^{2} \\
& =\bar{v}(x, y)+C_{1} y^{2}+C_{2},
\end{aligned}
$$

for all $(x, y, z) \in(0, \infty) \times \mathbb{R} \times\{0,1\}$, where $\bar{v}(x, y)$ is the post-default value function defined in (3.4), and the two constants $C_{1}>0$ and $C_{2}>R_{\alpha}$ are defined in Proposition 3.1. Here we switch to study sensitivity of these bounds with respect to the parameters WEA, SF, and DF. Figures 4 and 5 exhibit the relationships between the PDV and WEA, and the PDV, DF, and WEA, respectively. Figure 6 depicts the lower bound (B.2) and the upper bound (B.3) of the value function $\hat{v}$ with respect to the parameters SF and WEA.

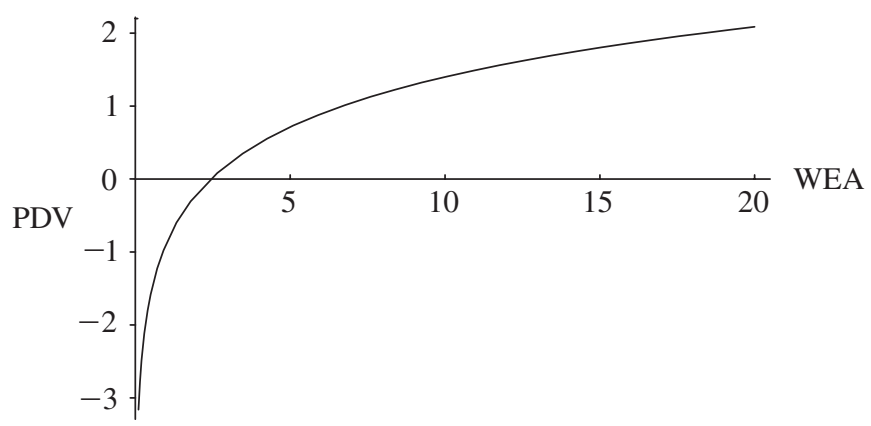

Figure 4: The PDV versus the WEA with DF $\alpha=\varepsilon+2 \bar{C}$.

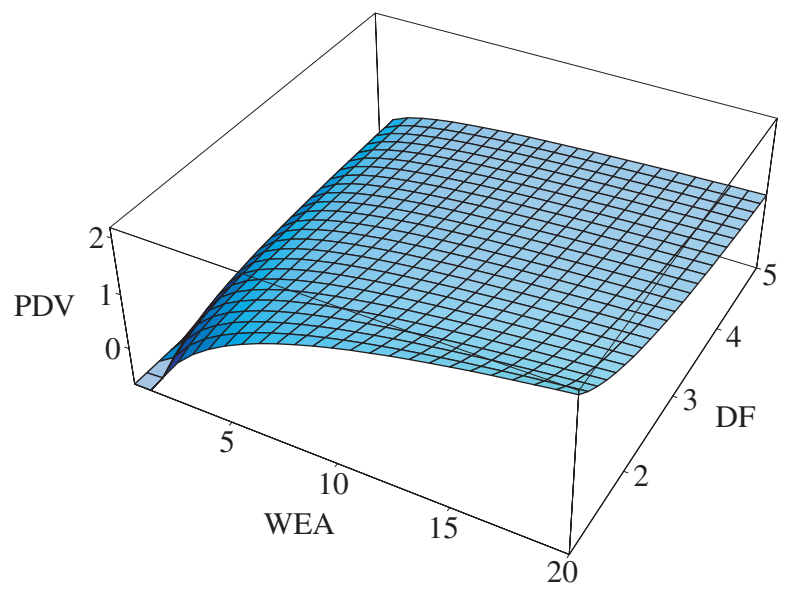

FIGURE 5: The PDV versus the DF and WEA. 


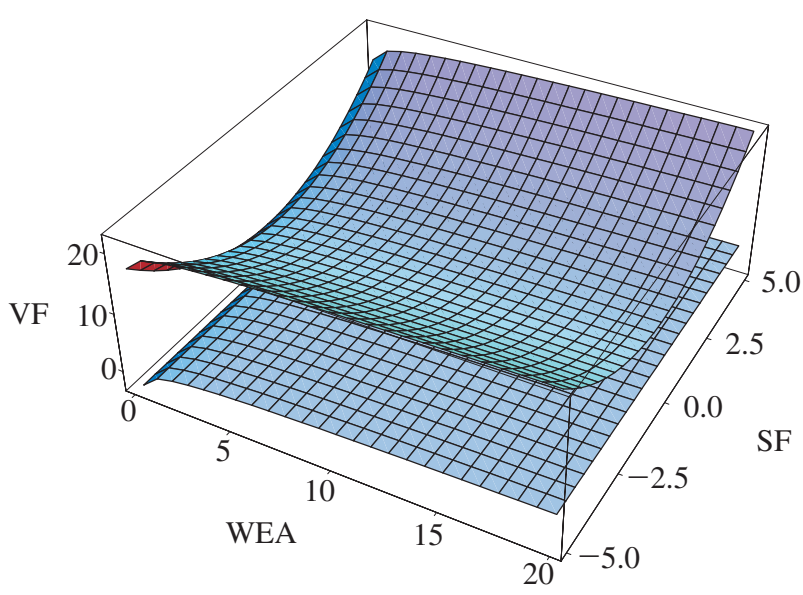

FIGURE 6: Upper and lower bounds for the of VF versus SF and WEA, with $C_{2}>R_{\alpha}$ and DF $\alpha=\varepsilon+2 \bar{C}$.

\section{Acknowledgements}

Research supported by the LPMC at Nankai University and the Key Grant Project of the Chinese Ministry of Education (no. 309009). The research of Bo and Yang was also supported by the Fundamental Research Funds for the Central Universities of China (granted by Xidian University, no. JY10000970002).

We thank the anonymous referees for their valuable comments and suggestions on an earlier version of this paper.

\section{References}

[1] Biagini, F. and Cretarola, A. (2006). Local risk-minimization for defaultable claims with recovery process. Preprint. LMU University of München and University of Bologna.

[2] Biagini, F. and Cretarola, A. (2007). Quadratic hedging methods for defaultable claims. Appl. Math. Optimization 56, 425-443.

[3] Biagini, F. and Cretarola, A. (2009). Local risk minimization for defaultable markets. Math. Finance 19, 669-689.

[4] Bielecki, T. R. ANd JANG, I. (2006). Portfolio optimization with a defaultable security. Asia-Pacific Financial Markets 13, 113-127.

[5] Bielecki, T. R. ANd Rutkowski, M. (2002). Credit Risk: Modelling, Valuation and Hedging. Springer, Berlin.

[6] Cox, J. C. AND Huang, C.-F. (1989). Optimal consumption and portfolio policies when asset prices follow a diffusion process. J. Econom. Theory 49, 33-83.

[7] Dellacherie, C. And Meyer, P.-A. (1982). Probabilities and Potential. B. North Holland, Amsterdam.

[8] Duffie, D. (2001). Dynamic Asset Pricing Theory, 3rd edn. Princeton University Press.

[9] Duffie, D. And Singleton, K. J. (1999). Modeling term structures of defaultable bonds. Rev. Financial Studies 12, 687-720.

[10] Fleming, W. H. and Hernández-Hernández, D. (2003). An optimal consumption model with stochastic volatility. Finance Stoch. 7, 245-262.

[11] Fleming, W. H. And Pang, T. (2004). An application of stochastic control theory to financial economics. SIAM J. Control Optimization 43, 502-531.

[12] Heston, S. L. (1993). A closed-form solution for options with stochastic volatility with applications to bond and currency options. Rev. Financial Studies 6, 327-343.

[13] IshiKawa, Y. (2004). Optimal control problem associated with jump processes. Appl. Math. Optimization 50, 21-65.

[14] JANG, I. (2005). Portfolio optimization with defaultable securities. Doctoral Thesis, University of Illinois at Chicago. 
[15] Jarrow, R. A., LANDo, D. AND Yu, F. (2005). Default risk and diversification: theory and empirical applications. Math. Finance 51, 1-26.

[16] Jin, X. AND Hou, Y. (2002). Optimal investment with default risk. FAME research paper no. 46, Switzerland.

[17] Karatzas, I., Lehoczky, J. P. And Shreve, S. E. (1987). Optimal portfolio and consumption decisions for a 'small investor' on a finite horizon. SIAM J. Control Optimization 27, 1557-1586.

[18] Korn, R. And Kraft, H. (2003). Optimal portfolios with defaultable securities: a firm value approach. Internat. J. Theory Appl. Finance 6, 793-819.

[19] Merton, R. C. (1969). Lifetime portfolio selection under uncertainty: the continuous case. Rev. Econom. Statist. $\mathbf{5 1 , 2 4 7 - 2 5 7 . ~}$

[20] Merton, R. C. (1971). Optimal consumption and portfolio rules in a continuous-time model. J. Econom. Theory 3, 373-413.

[21] Merton, R. C. (1992). Continuous-Time Finance. Blackwell, Oxford.

[22] PANG, T. (2006). Stochastic portfolio optimization with log utility. Internat. J. Theory Appl. Finance 9, 869-887.

[23] Pham, H. (2002). Smooth solutions to optimal investment methods with stochastic volatilities and portfolio constraints. Appl. Math. Optimization 46, 55-78.

[24] Protter, P. (2004). Stochastic Integration and Differential Equations, 2nd edn. Springer, Berlin. 